\title{
Dual HER-2 blockade therapy increases the risk of developing cardiac toxicities in HER-2 positive breast cancer: an up-to-date comprehensive meta-analysis
}

\author{
wei-xiang $\mathrm{Qi}^{1}$, Lu $\mathrm{Cao}^{1}$, Cheng Xu ${ }^{1}$, Shengguang Zhao ${ }^{1}$, and Jiayi Chen ${ }^{1}$ \\ ${ }^{1}$ Shanghai Jiao Tong University Medical School Affiliated Ruijin Hospital
}

June 24, 2020

\begin{abstract}
Background To investigate the incidence and risk of cardiac toxicities between dual HER-2 blockade and anti-HER-2 monotherapy. Materials and methods We searched PubMed, EMBASE and Cochrane library databases to identify relevant trials between January 11990 and October 31 2019. Statistical analyses were conducted to calculate the summary incidence, Petro odds radio (Peto ORs) and $95 \%$ confidence intervals (CIs) by using either random-effects or fixed-effects models. Results A total of 16,375 patients from 15 randomized controlled trials were included for analysis; the pooled incidence of LVEF decline and CHF in dual HER-2 blocked were $4.6 \%$ and $0.9 \%$, which was higher than that in anti-HER-2 monotherapy (3.2\% and $0.7 \%$, respectively). Dual HER-2 blockade therapy in breast cancer patients significantly increased the risk of developing LVEF decline (OR:1.19, 95\%CI: 1.02-1.40, $\mathrm{p}=0.031$ ) and CHF (OR:1.45, 95\%CI: 1.00-2.11, $\mathrm{p}=0.049$ ) when compared to anti-HER2 monotherapy. Subgroup analysis showed that addition of dual HER-2 blockade to adjuvant treatment for breast cancer significantly increased the risk of developing LVEF decline $(\mathrm{p}=0.048)$ and CHF $(\mathrm{p}=0.005)$. In addition, dual HER-2 blockade in breast cancer patients significantly increased the risk of developing LVEF decline $(\mathrm{p}=0.004)$ when compared to lapatinib alone, but not for CHF $(\mathrm{p}=0.11$, respectively). Conclusion Dual HER-2 targeted therapy in HER-2 positive breast cancer significantly increase the risk of developing LVEF and CHF when compared to anti-HER-2 alone, though the overall incidence of cardiac toxicities is very low. Physicians should be aware of this risk and provide close monitoring during the administration of dual HER-2 targeted therapy.
\end{abstract}

\section{Background}

To investigate the incidence and risk of cardiac toxicities between dual HER-2 blockade and anti-HER-2 monotherapy.

\section{Materials and methods}

We searched PubMed, EMBASE and Cochrane library databases to identify relevant trials between January 11990 and October 31 2019. Statistical analyses were conducted to calculate the summary incidence, Petro odds radio (Peto ORs) and 95\% confidence intervals (CIs) by using either random-effects or fixed-effects models.

\section{Results}

A total of 16,375 patients from 15 randomized controlled trials were included for analysis; the pooled incidence of LVEF decline and CHF in dual HER-2 blocked were $4.6 \%$ and $0.9 \%$, which was higher than that in antiHER-2 monotherapy (3.2\% and 0.7\%, respectively). Dual HER-2 blockade therapy in breast cancer patients significantly increased the risk of developing LVEF decline (OR:1.19, 95\%CI: 1.02-1.40, $p=0.031$ ) and CHF (OR:1.45, 95\%CI: 1.00-2.11, $p=0.049$ ) when compared to anti-HER2 monotherapy. Sub-group analysis 
showed that addition of dual HER-2 blockade to adjuvant treatment for breast cancer significantly increased the risk of developing LVEF decline $(p=0.048)$ and CHF $(p=0.005)$. In addition, dual HER-2 blockade in breast cancer patients significantly increased the risk of developing LVEF decline $(p=0.004)$ when compared to lapatinib alone, but not for $\mathrm{CHF}$ ( $p=0.11$, respectively).

\section{Conclusion}

Dual HER-2 targeted therapy in HER-2 positive breast cancer significantly increase the risk of developing LVEF and CHF when compared to anti-HER-2 alone, though the overall incidence of cardiac toxicities is very low. Physicians should be aware of this risk and provide close monitoring during the administration of dual HER-2 targeted therapy.

\section{Keywords: dual HER2 blockade; cancer; cardiac toxicities; meta-analysis;}

\section{Introduction}

During the past decades, the increased knowledge of biological mechanisms involving tumor proliferation and progression has led to the introduction of novel approaches for the treatment of cancer. The epidermal growth factor receptor (EGFR) family of transmembrane receptor tyrosine kinases (TKIs) is one such therapeutic target, which is overexpressed or mutated in multiple cancers $[1,2]$. Unlike other members of EGFR family, HER2/ErbB2 is a non-ligand-binding member of this family and exerts its activity through heterodimerization with other EGFR family members(EGFR, HER3,HER4)[3, 4]. And HER2 overexpression could also lead to HER2 dimerization and constitutive activation in the absence of ligand. Initially, HER2 overexpression is found in approximately $15-20 \%$ of breast cancer[5], then its overexpression was also detected in a subsets of many other cancer types, such as gastric[6], lung[7] or colorectal cancer[8]. In the last two decades, the introduction of monoclonal antibodies (MoAbs), TKIs or antibody-drug conjugates, which directly targets HER2 gene, has impressively improved the outcomes of HER-2 positive breast cancer patient in all disease stages[9-11]. Subsequently, the addition of anti-HER2 MoAbs to chemotherapy in HER-2 positive locally advanced gastric or gastro-oesophageal junction (GEJ) cancer also significantly improve overall survival. Currently, routine assessment of HER2 status in these tumors is mandatory, and the use of anti-HER2 agents have become a standard treatment for in HER-2 positive breast or gastric or GEJ cancer.

However, nearly all patients with metastatic HER2-positive breast cancer or gastric or GEJ cancer would eventually progress on anti-HER 2 therapy due to de novo or acquired resistance, potentially due to incomplete blockade of HER2 pathway. Therefore, inhibiting the HER2 signaling pathway more effectively with dual blockade approaches by using improved anti-HER2 therapies has been extensively investigated in both breast cancer and gastric or GEJ cancer, and dual HER2 blockade shows promising results in HER-2 positive breast cancer. Nevertheless, with the increasing use of dual HER2 blockade, particularly in the curative adjuvant setting, concerns regarding cardiac toxicities associated with dual anti-HER2 treatment in breast cancer patients has been increased. Therefore, there is an urgent need to clearly determine the overall incidence and risk of cardiac toxicities associated with dual HER2 blockade. Prior to the present study, Valachis $\mathrm{A}[12]$. performed a meta-analysis to investigate the risk of cardiac toxicities related to dual HER2 blockade in breast cancer, and found that cardiac toxicities in anti-HER2 combination therapy was comparable to that of anti-HER2 monotherapy[12]. But the sample size in that study are relative small, and most of the included studies have updated cardiac toxicities data. In addition, more randomized controlled trials assessing efficacy and toxicities of dual HER2 blockade in breast cancer have been conducted since then. As a result, we perform the present study to comprehensively investigate the incidence and risk of cardiac toxicities associated with dual HER-2 blockade in breast cancer when compared to anti-HER2 monotherapy by using a meta-analysis.

\section{Materials and methods}

\section{Study design}


We performed this systematic review and meta-analysis according to the Preferred Reporting Items for Systematic Reviews and Meta-Analyses statement guidelines 2009[13].

\section{Data source}

We conducted an independent review of citations from PubMed, EMBASE and Cochrane library databases between January 11990 and October 31 2019. Keywords were trastuzumab, lapatinib, pertuzumab, T-DM1, neratinib, pyrotinib, anti-HER2 agents, HER2 blockade, breast cancer, breast carcinoma, clinical trial and randomized. The search was limited to prospective randomized clinical trials published in English. The search strategy also used text terms such as anti-HER2 agents and HER-2 blockade to identify relevant information. We also performed independent searches using EMBASE and Cochrane library databases between January 11990 and October 31 2019, to identify relevant trials. Additionally, we searched abstracts containing the term "trastuzumab", "lapatinib", "T-DM1", "pertuzumab","anti-HER2 agents" that were presented at the American Society of Clinical Oncology (ASCO) and the European Society of Medical Oncology (ESMO) annual meetings from 2004 to 2013 to identify relevant studies. Each trials was carefully reviewed and in cases of duplicate publication only the most complete, recent and updated report of the clinical trial was included for analysis.

\section{Outcome definition}

The primary endpoints for the present study were left ventricular ejection fraction (LVEF) decline and congestive heart failure (CHF); LVEF decline was defined as LVEF decline less than $50 \%$ or a decrease of more than $10 \%$ from baseline, while CHF defined as New York Heart Association (NYHA) class III or IV or cardiac death. To avoid loss of information, trials reported LVEF decline more than $15 \%$ or $20 \%$ from baseline was also regarded as LVEF decline in the present analysis.

\section{Data extraction}

Data extraction was conducted independently by two investigators (WXQ and JYC), and any discrepancy between the reviewers was resolved by consensus. For each study, the following information was extracted: study name, treatment setting, anti-HER2 regimen and dosage, duration of anti-HER2 therapy, concomitant therapy, No. of LVEF decline, No. of CHF and No. of patients for safety analysis.

\section{Study selection}

Clinical trials that met the following criteria were included for analysis : (i) prospective, randomized, controlled phase 2 or 3 trials involving breast cancer patients; (ii) random assignment of participants to dual HER2 blockade or anti-HER2 monotherapy (lapatinib or trastuzumab or pertuzumab or T-DM1) with or without concomitant therapy in cancer patients regardless of treatment setting and (iii) available data regarding events or incidence of cardiac events and sample size. For trials with multiple intervention arms (for example, three-arms trials with two anti-HER2 monotherapy arms and one combined anti-HER2 therapy arm), we merged the two relevant (anti-HER2 monotherapy) arms into one group, then compared the merged group with dual HER-2 blockade group as previously reported[12]. Additionally, we used the five-item Jadad scale including randomization, double-blinding, and withdrawals as previously described to assess the quality of included trials. the final score was reported between 0 and 5[14].

\section{Statistical analysis}

The incidence and corresponding $95 \%$ confidence intervals (CIs) was calculated by the number of patients experiencing cardiac toxicities (LVEF decline or CHF) and total number of breast cancer patients treated with anti-HER2 agents were extracted from the safety profiles of included trials. Odds ratio (OR) and corresponding 95\% CIs were used to assessed the risk of cardiac toxicities associated with dual HER-2 blockade versus anti-HER-2 monotherapy. We used the Peto method to calculate the ORs and 95\% CIs because this method provided the best CI coverage and was more appropriate method for meta-analysis when dealing with low event $\operatorname{rates}(<5 \%)[15]$. To determine the risk of cardiac toxicities associated with dual anti-HER2 blockade within particular groups, we also conducted the following prespecified subgroup 
analyses: the treatment setting (neoadjuvant, adjuvant and metastatic), the type of anti-HER2 therapies (dual HER2 blockade vs. trastuzumab; dual HER2 blockade vs. lapatinib), concomitant treatment (none, chemotherapy and hormonal therapy). If Peto $\mathrm{OR}>1$, it reflected that more risk in dual HER2 blockade group, and vice versa. A statistical test with a $p$-value less than 0.05 was considered significant. To measure overall heterogeneity across the included cohorts, we calculated the $I^{2}$ statistic, with ${ }^{2}$ greater than $50 \%$ indicating high heterogeneity. Sensitivity analysis using different statistical models was performed to assess the stability of results. We assessed potential publication bias by visual inspection of the symmetry of funnel plots and with the Egger regression asymmetry test. All statistical analyses were performed by using Version 2 of the Comprehensive MetaAnalysis program (Biostat, Englewood, NJ) and Open Meta-Analyst software version 4.16.12 (Tufts University).

\section{Results}

\section{Search results}

A total of 470 studies were identified from the database search, 338 did not meet the inclusion criteria and were therefore excluded. Of the 132 studies were retrieved for full-text evaluation, a total of 22 trials met the inclusion criteria[16-37]. Six trials were update reports of published trials[17, 25, 26, 28, 29, 33], one trial involving gastric or GEJ carcinoma[18]. Thus finally 15 randomized controlled trials were included in this meta-analysis (figure 1). Table 1 listed the baseline characteristics of patients and studies. The quality of each included study was roughly assessed according to Jadad scale, four trials[16, 21, 34, 37] were doubleblind, placebo-controlled trials, thus had a Jadad score of 5 , and the remaining eleven trials were open-label controlled trials, thus had a Jadad score of 3 .

\section{Incidence of LVEF decline}

A total of 7,184 patients receiving dual HER-2 blockade in 15 RCTs were available for LVEF decline analysis. There were $302 \mathrm{LVEF}$ decline events among these patients. The highest incidence $(8.7 \%$; $95 \%$ CI $5.3 \%$ to $13.9 \%$ ) was observed in a phase III breast cancer neoadjuvant trial of trastuzumab plus lapatinib when concomitant with paclitaxel [22], while the lowest incidence was observed in four trials in which no events of LVEF decline occurred[16, 27, 31, 37]. Using a random-effects model $(\chi 2$-based $\mathrm{Q}$ statistic test: $\mathrm{Q}=31.75$; $p=0.004 ; I^{2}=55 \%$ ), the summary incidence of LVEF decline in cancer patients treated with dual HER2 blockade was $4.6 \%$ (95\% CI, $3.7 \%$ to $5.7 \%$, figure $2 \mathrm{~A}$ ).

For LVEF decline associated with anti-HER2 monotherapy, the highest incidence $(13.4 \%$; $95 \%$ CI $10.2 \%$ to $17.4 \%$ ) was observed in NSABP protocol B-41 trial[30], while no events of LVEF decline occurred in three trials[16, 19, 37]. Using a random-effects model $\left(\chi 2\right.$-based $\mathrm{Q}$ statistic test: $\left.\mathrm{Q}=108.33 ; p<0.001 ; I^{2}=86 \%\right)$, the summary incidence of LVEF decline in cancer patients treated with anti-HER2 monotherapy was $3.2 \%$ (95\% CI, $2.2 \%$ to $4.6 \%$, figure $2 \mathrm{~B}$ ).

\section{Incidence of CHF}

A total of 6,818 patients receiving dual HER-2 blockade in 14 RCTs were available for CHF analysis. There were 56 total CHF events among these patients. The highest incidence $(2.0 \%$; 95\% CI 0.7 to $6.1 \%)$ was observed in a phase III breast cancer trial of trastuzumab plus lapatinib after prior trastuzumab-based therapies[33], while no events of CHF occurred in five trials. Using a fixed-effects model ( $\chi 2$-based Q statistic test: $\left.\mathrm{Q}=8.26 ; p=0.82 ; I^{2}=0 \%\right)$, the summary incidence of CHF in cancer patients treated with dual HER2 blockade was $0.9 \%$ (95\% CI, $0.7 \%$ to $1.2 \%$, figure $2 \mathrm{C}$ ). As for CHF associated with anti-HER 2 monotherapy, a total of 9,283 patients were included for analysis. Using a random-effects model ( $\chi 2$-based $\mathrm{Q}$ statistic test: $\left.\mathrm{Q}=52.77 ; p<0.001 ; I^{2}=73.42 \%\right)$, the summary incidence of CHF in cancer patients treated with antiHER2 monotherapy was $0.7 \%$ (95\% CI, $0.4 \%$ to $1.3 \%$, figure 2D).

\section{Risk of LVEF decline and CHF associated with dual HER2 blockade}

All of the 15 included trials reported the LVEF decline data, thus included for calculating the OR of LVEF decline associated with dual HER2 blockade. A total of 284 LVEF decline events were observed in dual HER2 
blockade versus 357 LVEF decline events in anti-HER2 monotherapy. The pooled results demonstrated that the dual HER-2 blockade in cancer patients significantly increased the risk of developing LVEF decline with an OR of 1.20 (95\% CI 1.02-1.41, $p=0.031$, Figure 3A) using a fixed-effects model ( $\chi 2$-based $\mathrm{Q}$ statistic test: $\left.\mathrm{Q}=22.58 ; p=0.091, I^{2}=32.6\right)$.

A total of 14 randomized trials reported CHF data. A total of 55 CHF events were observed in dual HER2 blockade versus $57 \mathrm{CHF}$ events in anti-HER2 monotherapy. The pooled results showed that the dual HER2 blockade in cancer patients significantly increased risk of developing CHF with an OR of 1.45 (95\% CI $1.00-2.11, p=0.049$, Figure $3 \mathrm{~B})$ using a fixed-effects model $\left(\chi^{2}\right.$-based $\mathrm{Q}$ statistic test: $\mathrm{Q}=12.67 ; p=0.53, I$ $2=0)$.

\section{Sensitivity analysis}

Sensitivity analyses using Mantel Haenszel or Inverse Variance model showed that the risk of LVEF decline with dual HER-2 blockade was 1.19(95\%CI: 1.02-1.41, $p=0.031$ ) and 1.18 (95\%CI: $1.01-1.40, p=0.041)$. Similarly, sensitivity analyses using Mantel Haenszel or Inverse Variance model showed that the risk of CHF associated with dual HER-2 blockade was 1.48 (95\%CI: 1.01-2.16, $p=0.045)$ and 1.55(95\%CI: 1.04-2.25, $p$ $=0.032$ ) (supplemental table 1) when compared to anti-HER2 monotherapy.

\section{Sub-group analysis}

Firstly, we carried out a subgroup risk analysis stratified according to treatment settings. Our results demonstrated that risk of LVEF decline and CHF was comparable between dual HER-2 blockade and antiHER2 monotherapy in neoadjuvant or metastatic setting (both $p>0.05$, table 2 ). However, the addition of dual HER2 blockade to adjuvant treatment in breast cancer significantly increased the risk of developing CHF (OR 2.00, 95\%CI: 1.23-3.24, $p=0.005$ ) and LVEF decline (OR1.17, 95\%CI: 1.00-1.38, $p=0.048$, table 2).

The concomitant treatment with anti-HER-2 therapy might impact the ORs of cardiac toxicities. Our combined results demonstrated that concomitant hormonal therapy with dual HER2 blockade in breast cancer significantly increased risk of developing LVEF decline in comparison with hormonal therapy plus antiHER2 monotherapy (OR 4.51, 95\%CI:1.24-16.40, $p=0.022$ ), while no concomitant treatment or concomitant chemotherapy with dual HER-2 blockade did not increase the risk of developing LVEF decline $(p=0.19$ and $p=0.11$, respectively). As for CHF events, concomitant chemotherapy with dual HER2 blockade treatment significantly increased the risk of developing CHF when compared to anti-HER2 monotherapy plus chemotherapy (OR 1.47, 95\%CI: 1.00-2.16, $p=0.052$ ), while no concomitant treatment or concomitant hormonal therapy with dual HER-2 blockade did not increase the risk of CHF ( $p=0.22$ and $p=0.59$, respectively).

We also did sub-group analysis according to anti-HER-2 monotherapy. Our result showed that dual HER-2 blockade significantly increased the risk of developing LVEF decline (OR 1.49, 95\%CI: 1.14-1.96, $p=0.004$ ) when compared to lapatinib, but not for CHF (OR 2.62, 95\%CI: 0.90-2.94, $p=0.11$ ). In comparison with trastuzumab alone, no significantly increased risk of developing $\operatorname{LVEF}$ decline $(p=0.37)$ and CHF $(p=0.24$, table 2) was observed in dual HER-2 blockade group.

\section{Publication Bias}

We performed Begg's funnel plot and Egger's test to detect the publication bias of literatures. No significant evidence of publication bias for LVEF decline and CHF was detected by using funnel plots (supplemental figure 1 for LVEF decline; and supplemental figure 2 for CHF), Begg's test (LVEF decline, $p=0.62$; CHF, $p=0.53$ ) and Egger's test (LVEF decline, $p=0.38$; CHF, $p=0.93$ ).

\section{Discussion}

To our best knowledge, this meta-analysis is the largest and most comprehensive meta-analysis to assess the risk of cardiac toxicities associated with dual HER2 blockade versus anti-HER2 monotherapy. A total of 16,375 patients from 15 randomized controlled trials were included for analysis. Our pooled results indicate 
that the overall incidence rate of LVEF decline and CHF in dual HER-2 blockade are $4.6 \%$ and $0.9 \%$, which is higher than that of anti-HER-2 monotherapy ( $3.2 \%$ and $0.7 \%$, respectively). Importantly, the present study for the first time demonstrates that dual HER-2 blockade therapy in breast cancer patients increases $19 \%$ risk of developing LVEF decline and $45 \%$ risk of developing CHF when compared to anti-HER2 monotherapy. It should be noted that patients with inadequate cardiac function would be excluded from treatment and close cardiac monitoring has been performed during the administration of anti-HER-2 agents, both of them would significantly reduced the incidence of cardiac toxicities. Although the overall incidence of cardiac toxicities is very low, a slight but significant risk of developing cardiac toxicities has been observed in dual HER-2 blockade when compared to anti-HER-2 monotherapy.

Sub-group analysis showed that addition of dual HER-2 blockade to adjuvant treatment in breast cancer significantly increased the risk of developing LVEF decline $(p=0.031)$ and CHF $(p=0.049)$, but not for neoadjuvant or metastatic settings. Additionally, cardiac toxicities associated with specific anti-HER-2 agents might be difference, which might be attributable to the unique epitopes of HER2 recognized by each antibody and differential effects on downstream signaling pathways[38]. The cardiac toxicities associated with trastuzumab seems higher than that of lapatinib. In a previous publication based on 29,000 breast cancer, the authors found that incidence severe cardiac toxicities with trastuzumab was 3.0\%(95\% CI: 2.413.64)[39], while the overall cardiac toxicities associated with lapatinib was 3.0\%[40]. We therefore perform sub-group analysis based on anti-HER-2 agents, and find that dual HER-2 blockade in breast cancer patients significantly increased the risk of developing LVEF decline $(p=0.004)$ when compared to lapatinib alone, but not for CHF ( $p=0.11$, respectively). No significant difference of cardiac toxicities is found between dual HER-2 blockade and trastuzumab. Additionally, the concomitant hormonal treatment with dual HER-2 blockade in breast cancer significantly increases the risk of developing LVEF decline in comparison with hormonal therapy plus anti-HER2 monotherapy $(p=0.022)$, while concomitant chemotherapy with dual HER2 blockade treatment also significantly increases the risk of developing CHF when compared to antiHER2 monotherapy plus chemotherapy $(p=0.052)$. Based on our finding, dual HER-2 blockade therapy in breast cancer is associated with a small but statistically significant risk of developing LVEF decline and CHF when compared with anti-HER2 monotherapy. In comparison with anti-HER-2 monotherapy, addition of dual HER-2 blockade to neoadjuvant or metastatic settings for breast cancer is safe in terms of cardiac toxicities, but not for adjuvant setting. Physicians should pay more attention to cardiac toxicities during the administration of dual HER-2 treatment when concomitant hormonal/chemotherapy treatment.

The molecular mechanisms related to cardiac toxicities induced by anti-HER2 agents, either alone or in combination, remains unknown. One potential explanation for the mechanism is the inhibition of NRG1/HER2 signaling pathway by using anti-HER-2 agents[41]. In multiple vitro studies have demonstrated that the NRG-1/ErbB2 signaling pathway plays an important role in controlling cardiomyocytes proliferation, survival and myofibril disarray in cardiomyocytes[38].

The main strengths of this study are that clinical trials are identified by a systematic literature review with the largest and comprehensive meta-analysis to investigate the cardiac toxicities associated with dual HER-2 blockade versus anti-HER-2 monotherapy. The quality of this evidence is high because all of the included trials are prospective randomized controlled trials. However, several limitations are needed to be concerned. First of all, patients enrolled in prospective trials generally need to have adequate cardiac function, and close cardiac monitoring is implemented for all treated patients, which might underestimate the incidence of cardiac toxicities in common oncology practice. Secondly, this is a meta-analysis of published data, and lack of individual patient data prevents us from adjusting the cardiac toxicities according to previous treatment and patient variables. For example, the cardiac safety of dual HER-2 blockade in breast cancer patients with preexisting cardiovascular risk factors remains undetermined. Finally, these studies are conducted at various international institutions by different investigators and may have potential bias in reporting the types of cardiac events. In addition, the primary endpoints of the these included studies are aimed to investigate the survival benefit of anti-HER2 therapy in cancer patients, but not for cardiac toxicities related dual HER-2 blockade. Thus, the frequency of cardiac toxicities might be underreported in clinical trials. 


\section{Conclusion}

Although the overall incidence of cardiac toxicities is very low, dual HER-2 targeted therapy in breast cancer significantly increase the risk of developing LVEF and CHF when compared to anti-HER-2 alone. Sub-group analysis that addition of dual HER-2 blockade to adjuvant treatment in breast cancer increased the risk of developing cardiac toxicities but not for neoadjuvant or metastatic settings. Concomitant treatment with dual HER2 blockade would increase the risk of developing cardiac toxicities. Physicians should be aware of this risk and provide close monitoring during the administration of dual HER-2 targeted therapy.

\section{Competing interests:}

All authors have completed the Unified Competing Interest form athttp://www.icmje.org/coi_disclosure.pdf (available on request from the corresponding author) and declare no support from any organization for the submitted work, no financial relationships with any organizations that might have an interest in the submitted work in the previous 3 years and no other relationships or activities that could appear to have influenced the submitted work.

We are indebted to the authors of the primary studies, for without their contributions, this work would have been impossible.

Funding : none

\section{References:}

1. Sigismund S, Avanzato D, Lanzetti L: Emerging functions of the EGFR in cancer . Molecular oncology 2018, 12 (1):3-20.

2. Seshacharyulu P, Ponnusamy MP, Haridas D, Jain M, Ganti AK, Batra SK: Targeting the EGFR signaling pathway in cancer therapy . Expert opinion on therapeutic targets 2012, 16 (1):15-31.

3. Graus-Porta D, Beerli RR, Daly JM, Hynes NE: ErbB-2, the preferred heterodimerization partner of all ErbB receptors, is a mediator of lateral signaling. The EMBO journal 1997, 16 (7):1647-1655.

4. Moasser MM: The oncogene HER2: its signaling and transforming functions and its role in human cancer pathogenesis . Oncogene 2007, 26 (45):6469-6487.

5. Seshadri R, Firgaira FA, Horsfall DJ, McCaul K, Setlur V, Kitchen P: Clinical significance of HER2/neu oncogene amplification in primary breast cancer. The South Australian Breast Cancer Study Group . Journal of clinical oncology : official journal of the American Society of Clinical Oncology 1993, 11 (10):1936-1942.

6. Nakajima M, Sawada H, Yamada Y, Watanabe A, Tatsumi M, Yamashita J, Matsuda M, Sakaguchi T, Hirao T, Nakano H: The prognostic significance of amplification and overexpression of c-met and c-erb B-2 in human gastric carcinomas .Cancer 1999, 85 (9):1894-1902.

7. Yoshizawa A, Sumiyoshi S, Sonobe M, Kobayashi M, Uehara T, Fujimoto M, Tsuruyama T, Date H, Haga H:HER2 status in lung adenocarcinoma: a comparison of immunohistochemistry, fluorescence in situ hybridization (FISH), dual-ISH, and gene mutations . Lung cancer 2014,85 (3):373-378.

8. La Salvia A, Lopez-Gomez V, Garcia-Carbonero R: HER2-targeted therapy: an emerging strategy in advanced colorectal cancer . Expert opinion on investigational drugs 2019, 28 (1):29-38.

9. Slamon DJ, Leyland-Jones B, Shak S, Fuchs H, Paton V, Bajamonde A, Fleming T, Eiermann W, Wolter J, Pegram Met al : Use of chemotherapy plus a monoclonal antibody against HER2 for metastatic breast cancer that overexpresses HER2. The New England journal of medicine 2001,344 (11):783-792.

10. Pivot X, Romieu G, Debled M, Pierga JY, Kerbrat P, Bachelot T, Lortholary A, Espie M, Fumoleau $\mathrm{P}$, Serin $\mathrm{D}$ et al : 6 months versus 12 months of adjuvant trastuzumab in early breast cancer 
(PHARE): final analysis of a multicentre, open-label, phase 3 randomised trial . Lancet 2019,393 (10191):2591-2598.

11. van Ramshorst MS, van der Voort A, van Werkhoven ED, Mandjes IA, Kemper I, Dezentje VO, Oving IM, Honkoop AH, Tick LW, van de Wouw AJ et al : Neoadjuvant chemotherapy with or without anthracyclines in the presence of dual HER2 blockade for HER2-positive breast cancer (TRAIN-2): a multicentre, open-label, randomised, phase 3 trial . The Lancet Oncology 2018,19 (12):1630-1640.

12. Valachis A, Nearchou A, Polyzos NP, Lind P: Cardiac toxicity in breast cancer patients treated with dual HER2 blockade. Int J Cancer 2013,133 (9):2245-2252.

13. Liberati A, Altman DG, Tetzlaff J, Mulrow C, Gotzsche PC, Ioannidis JP, Clarke M, Devereaux PJ, Kleijnen J, Moher D: The PRISMA statement for reporting systematic reviews and meta-analyses of studies that evaluate healthcare interventions: explanation and elaboration . $B m j 2009,339$ :b2700.

14. Moher D, Pham B, Jones A, Cook DJ, Jadad AR, Moher M, Tugwell P, Klassen TP: Does quality of reports of randomised trials affect estimates of intervention efficacy reported in meta-analyses? Lancet 1998,352 (9128):609-613.

15. Sweeting MJ, Sutton AJ, Lambert PC: What to add to nothing? Use and avoidance of continuity corrections in meta-analysis of sparse data . Statistics in medicine 2004, 23 (9):1351-1375.

16. Shao Z, Pang D, Yang H, Li W, Wang S, Cui S, Liao N, Wang Y, Wang C, Chang YC et al :Efficacy, Safety, and Tolerability of Pertuzumab, Trastuzumab, and Docetaxel for Patients With Early or Locally Advanced ERBB2-Positive Breast Cancer in Asia: The PEONY Phase 3 Randomized Clinical Trial .JAMA oncology 2019.

17. Perez EA, Barrios C, Eiermann W, Toi M, Im YH, Conte P, Martin M, Pienkowski T, Pivot XB, Burris HA, 3rdet al : Trastuzumab emtansine with or without pertuzumab versus trastuzumab with taxane for human epidermal growth factor receptor 2-positive advanced breast cancer: Final results from MARIANNE . Cancer 2019, 125 (22):3974-3984.

18. Tabernero J, Hoff PM, Shen L, Ohtsu A, Shah MA, Cheng K, Song C, Wu H, Eng-Wong J, Kim K et al :Pertuzumab plus trastuzumab and chemotherapy for HER2-positive metastatic gastric or gastro-oesophageal junction cancer (JACOB): final analysis of a double-blind, randomised, placebo-controlled phase 3 study . The Lancet Oncology 2018, 19 (10):1372-1384.

19. Rimawi M, Ferrero JM, de la Haba-Rodriguez J, Poole C, De Placido S, Osborne CK, Hegg R, Easton V, Wohlfarth C, Arpino G et al : First-Line Trastuzumab Plus an Aromatase Inhibitor, With or Without Pertuzumab, in Human Epidermal Growth Factor Receptor 2-Positive and Hormone Receptor-Positive Metastatic or Locally Advanced Breast Cancer (PERTAIN): A Randomized, Open-Label Phase II Trial . Journal of clinical oncology : official journal of the American Society of Clinical Oncology 2018,36 (28):2826-2835.

20. Johnston SRD, Hegg R, Im SA, Park IH, Burdaeva O, Kurteva G, Press MF, Tjulandin S, Iwata H, Simon SDet al : Phase III, Randomized Study of Dual Human Epidermal Growth Factor Receptor 2 (HER2) Blockade With Lapatinib Plus Trastuzumab in Combination With an Aromatase Inhibitor in Postmenopausal Women With HER2-Positive, Hormone ReceptorPositive Metastatic Breast Cancer: ALTERNATIVE . Journal of clinical oncology : official journal of the American Society of Clinical Oncology 2018,36 (8):741-748.

21. von Minckwitz G, Procter M, de Azambuja E, Zardavas D, Benyunes M, Viale G, Suter T, Arahmani A, Rouchet N, Clark E et al : Adjuvant Pertuzumab and Trastuzumab in Early HER2-Positive Breast Cancer. The New England journal of medicine 2017, 377 (2):122-131. 
22. Urruticoechea A, Rizwanullah M, Im SA, Ruiz ACS, Lang I, Tomasello G, Douthwaite H, Badovinac Crnjevic T, Heeson S, Eng-Wong J et al : Randomized Phase III Trial of Trastuzumab Plus Capecitabine With or Without Pertuzumab in Patients With Human Epidermal Growth Factor Receptor 2-Positive Metastatic Breast Cancer Who Experienced Disease Progression During or After Trastuzumab-Based Therapy. Journal of clinical oncology : official journal of the American Society of Clinical Oncology 2017,35 (26):3030-3038.

23. Perez EA, Barrios C, Eiermann W, Toi M, Im YH, Conte P, Martin M, Pienkowski T, Pivot X, Burris H, 3rdet al : Trastuzumab Emtansine With or Without Pertuzumab Versus Trastuzumab Plus Taxane for Human Epidermal Growth Factor Receptor 2-Positive, Advanced Breast Cancer: Primary Results From the Phase III MARIANNE Study . Journal of clinical oncology : official journal of the American Society of Clinical Oncology 2017,35 (2):141-148.

24. Piccart-Gebhart M, Holmes E, Baselga J, de Azambuja E, Dueck AC, Viale G, Zujewski JA, Goldhirsch A, Armour A, Pritchard KI et al : Adjuvant Lapatinib and Trastuzumab for Early Human Epidermal Growth Factor Receptor 2-Positive Breast Cancer: Results From the Randomized Phase III Adjuvant Lapatinib and/or Trastuzumab Treatment Optimization Trial . Journal of clinical oncology : official journal of the American Society of Clinical Oncology 2016, 34 (10):1034-1042.

25. Gianni L, Pienkowski T, Im YH, Tseng LM, Liu MC, Lluch A, Staroslawska E, de la Haba-Rodriguez J, Im SA, Pedrini JL et al : 5-year analysis of neoadjuvant pertuzumab and trastuzumab in patients with locally advanced, inflammatory, or early-stage HER2-positive breast cancer (NeoSphere): a multicentre, open-label, phase 2 randomised trial . The Lancet Oncology 2016, 17 (6):791-800.

26. Swain SM, Baselga J, Kim SB, Ro J, Semiglazov V, Campone M, Ciruelos E, Ferrero JM, Schneeweiss A, Heeson S et al : Pertuzumab, trastuzumab, and docetaxel in HER2-positive metastatic breast cancer. The New England journal of medicine 2015, 372 (8):724-734.

27. Bonnefoi H, Jacot W, Saghatchian M, Moldovan C, Venat-Bouvet L, Zaman K, Matos E, Petit T, Bodmer A, Quenel-Tueux N et al : Neoadjuvant treatment with docetaxel plus lapatinib, trastuzumab, or both followed by an anthracycline-based chemotherapy in HER2-positive breast cancer: results of the randomised phase II EORTC 10054 study. Annals of oncology : official journal of the European Society for Medical Oncology / ESMO2015, 26 (2):325-332.

28. de Azambuja E, Holmes AP, Piccart-Gebhart M, Holmes E, Di Cosimo S, Swaby RF, Untch M, Jackisch C, Lang I, Smith I et al : Lapatinib with trastuzumab for HER2-positive early breast cancer (NeoALTTO): survival outcomes of a randomised, open-label, multicentre, phase 3 trial and their association with pathological complete response. The Lancet Oncology 2014,15 (10):1137-1146.

29. Swain SM, Kim SB, Cortes J, Ro J, Semiglazov V, Campone M, Ciruelos E, Ferrero JM, Schneeweiss A, Knott Aet al : Pertuzumab, trastuzumab, and docetaxel for HER2-positive metastatic breast cancer (CLEOPATRA study): overall survival results from a randomised, double-blind, placebo-controlled, phase 3 study . The Lancet Oncology 2013, 14 (6):461-471.

30. Robidoux A, Tang G, Rastogi P, Geyer CE, Jr., Azar CA, Atkins JN, Fehrenbacher L, Bear HD, BaezDiaz L, Sarwar S et al : Lapatinib as a component of neoadjuvant therapy for HER2-positive operable breast cancer (NSABP protocol B-41): an open-label, randomised phase 3 trial. The Lancet Oncology2013, 14 (12):1183-1192.

31. Guarneri V, Frassoldati A, Bottini A, Cagossi K, Bisagni G, Sarti S, Ravaioli A, Cavanna L, Giardina G, Musolino A et al : Preoperative chemotherapy plus trastuzumab, lapatinib, or both in human epidermal growth factor receptor 2-positive operable breast cancer: results of the randomized phase II CHER-LOB study. Journal of clinical oncology : official journal of the American Society of Clinical Oncology 2012,30 (16):1989-1995.

32. Gianni L, Pienkowski T, Im YH, Roman L, Tseng LM, Liu MC, Lluch A, Staroslawska E, de la Haba- 
Rodriguez J, Im SA et al : Efficacy and safety of neoadjuvant pertuzumab and trastuzumab in women with locally advanced, inflammatory, or early HER2-positive breast cancer (NeoSphere): a randomised multicentre, open-label, phase 2 trial . The Lancet Oncology2012, 13 (1):25-32.

33. Blackwell KL, Burstein HJ, Storniolo AM, Rugo HS, Sledge G, Aktan G, Ellis C, Florance A, Vukelja $\mathrm{S}$, Bischoff $\mathrm{J}$ et al : Overall survival benefit with lapatinib in combination with trastuzumab for patients with human epidermal growth factor receptor 2-positive metastatic breast cancer: final results from the EGF104900 Study. Journal of clinical oncology : official journal of the American Society of Clinical Oncology 2012, 30 (21):2585-2592.

34. Baselga J, Cortes J, Kim SB, Im SA, Hegg R, Im YH, Roman L, Pedrini JL, Pienkowski T, Knott A et al : Pertuzumab plus trastuzumab plus docetaxel for metastatic breast cancer. The New England journal of medicine 2012,366 (2):109-119.

35. Baselga J, Bradbury I, Eidtmann H, Di Cosimo S, de Azambuja E, Aura C, Gomez H, Dinh P, Fauria $\mathrm{K}$, Van Dooren V et al : Lapatinib with trastuzumab for HER2-positive early breast cancer (NeoALTTO): a randomised, open-label, multicentre, phase 3 trial . Lancet 2012,379 (9816):633640 .

36. Blackwell KL, Burstein HJ, Storniolo AM, Rugo H, Sledge G, Koehler M, Ellis C, Casey M, Vukelja $\mathrm{S}$, Bischoff J et al : Randomized study of Lapatinib alone or in combination with trastuzumab in women with ErbB2-positive, trastuzumab-refractory metastatic breast cancer . Journal of clinical oncology : official journal of the American Society of Clinical Oncology 2010, 28 (7):1124-1130.

37. Xu B. LW, Zhang Q., Z. Shao,X. Wang, H. Li, T. Sun, Y. Yin, H. Zheng, H. Zhang, T. Chan, G. Lei, E. Restuccia: A phase III, randomized, double-blind, placebo (Pla)-controlled study of pertuzumab (P) + trastuzumab $(\mathbf{H})+$ docetaxel $(\mathrm{D})$ versus Pla $+\mathbf{H}+\mathrm{D}$ in previously untreated HER2positive locally recurrent/metastatic breast cancer (LR/MBC) (PUFFIN). Journal of Clinical Oncology 2019, 37 (15_suppl):1026-1026

38. Fedele C, Riccio G, Malara AE, D'Alessio G, De Lorenzo C: Mechanisms of cardiotoxicity associated with ErbB2 inhibitors. Breast cancer research and treatment 2012, 134 (2):595-602.

39. Mantarro S, Rossi M, Bonifazi M, D'Amico R, Blandizzi C, La Vecchia C, Negri E, Moja L: Risk of severe cardiotoxicity following treatment with trastuzumab: a meta-analysis of randomized and cohort studies of $\mathbf{2 9 , 0 0 0}$ women with breast cancer. Internal and emergency medicine 2016,11 (1):123-140.

40. Choi HD, Chang MJ:Cardiac toxicities of lapatinib in patients with breast cancer and other HER2-positive cancers: a meta-analysis . Breast cancer research and treatment 2017, 166 (3):927-936.

41. Gassmann M, Casagranda F, Orioli D, Simon H, Lai C, Klein R, Lemke G: Aberrant neural and cardiac development in mice lacking the ErbB4 neuregulin receptor .Nature 1995, 378 (6555):390394.

Figure legends

Figure 1: Flow chart of study selection in the meta-analysis

Figure 2: incidence of LVEF and CHF associated with dual HER-2 blockade and anti-HER-2 monotherapy in breast cancer;

Figure 3: Risk of LVEF and CHF associated with dual HER-2 blockade versus anti-HER-2 monotherapy in breast cancer;

Supplemental figure 1: Funnel plot of publication bias for LVEF decline in the meta-analysis

Supplemental figure 2: : Funnel plot of publication bias for CHF in the meta-analysis 


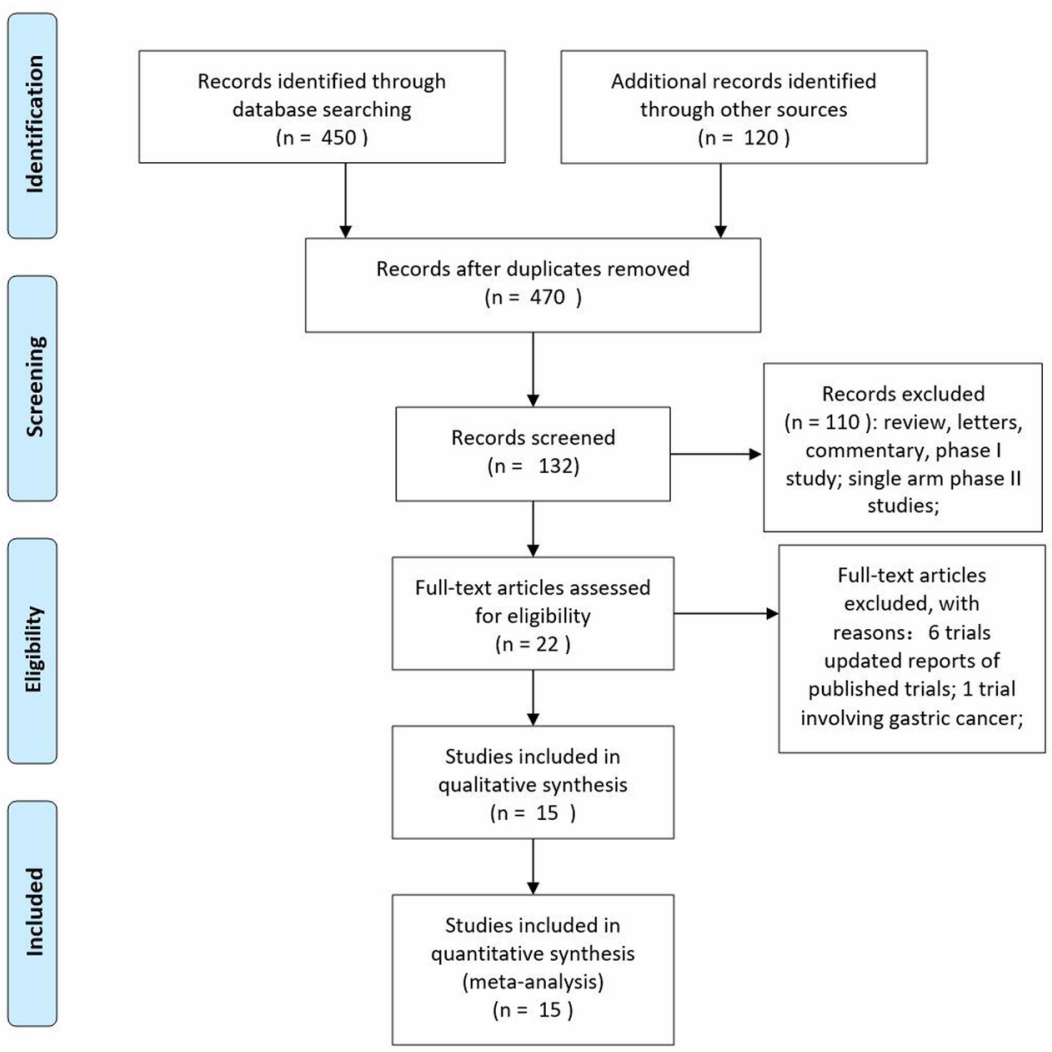



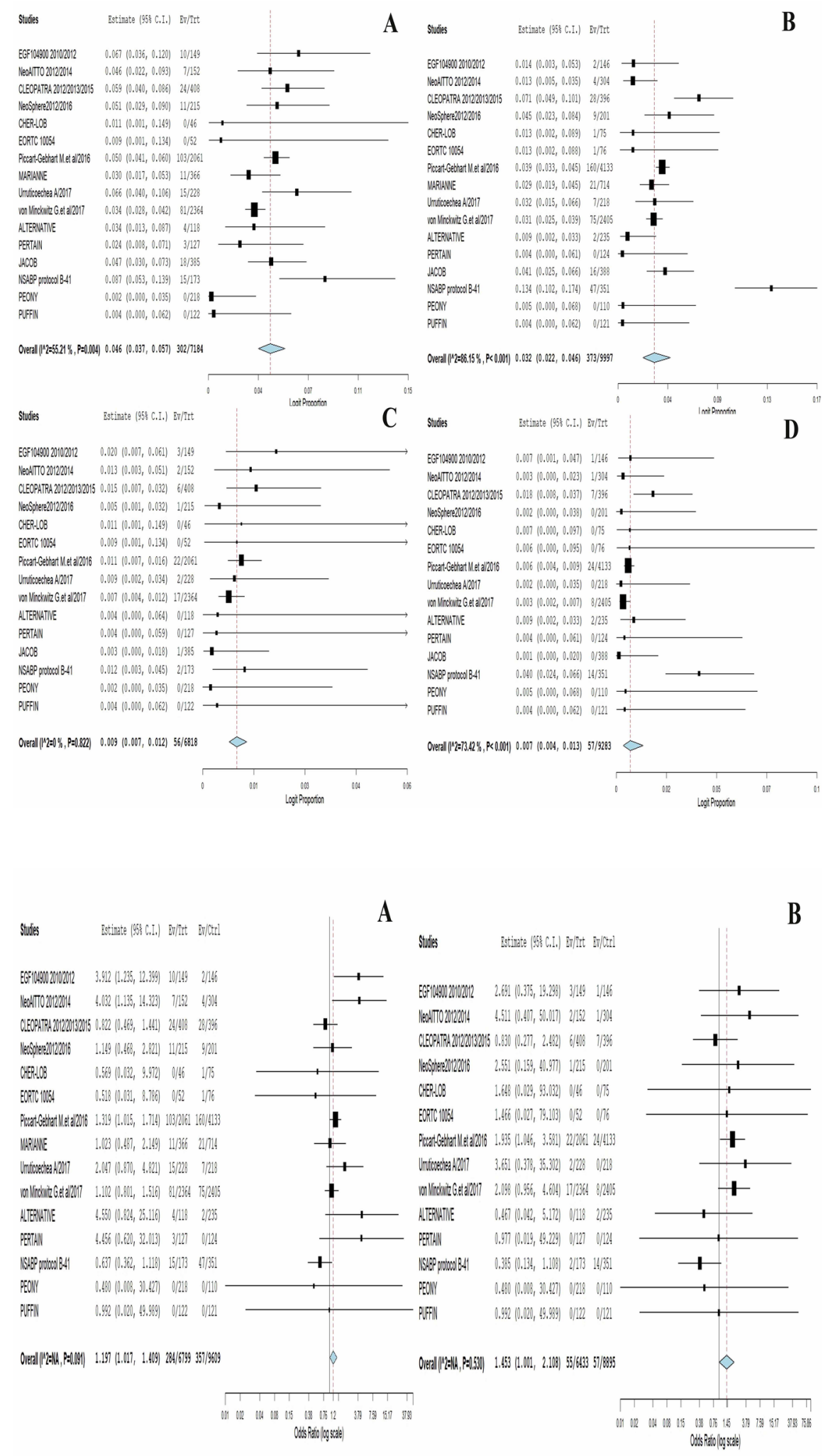


\section{Hosted file}

table 1.docx available at https://authorea.com/users/336386/articles/462102-dual-her-2blockade-therapy-increases-the-risk-of-developing-cardiac-toxicities-in-her-2-positivebreast-cancer-an-up-to-date-comprehensive-meta-analysis

\section{Hosted file}

table 2.docx available at https://authorea.com/users/336386/articles/462102-dual-her-2blockade-therapy-increases-the-risk-of-developing-cardiac-toxicities-in-her-2-positivebreast-cancer-an-up-to-date-comprehensive-meta-analysis 